\title{
A Polycyclic Aromatic Hydrocarbon for the Detection of Nonpolar Analytes under Counteracting Humidity Conditions
}

\begin{tabular}{|c|c|}
\hline Journal: & ACS Applied Materials \& Interfaces \\
\hline Manuscript ID: & am-2012-013328.R2 \\
\hline Manuscript Type: & Article \\
\hline Date Submitted by the Author: & 28-Aug-2012 \\
\hline Complete List of Authors: & $\begin{array}{l}\text { Bachar, Nadav; Technion - Israel Institute of Technology, Department of } \\
\text { Chemical Engineering } \\
\text { Mintz, Lena; Technion - Israel Institute of Technology, Department of } \\
\text { Chemical Engineering } \\
\text { Zilberman, Yael; Technion - Israel Institute of Technology, Department of } \\
\text { Chemical Engineering } \\
\text { Ionescu, Radu; Rovira i Virgili University, Department of Electronics, } \\
\text { Electrical and Automatic Engineering } \\
\text { Feng, Xinliang; Max-Planck Institute for Polymer Research, Synthetic } \\
\text { Chemistry } \\
\text { Mullen, Klaus; Max-Planck-Institute for Polymer Research, } \\
\text { Haick, Hossam; Technion - Israel Institute of Technology, Department of } \\
\text { Chemical Engineering }\end{array}$ \\
\hline
\end{tabular}




\title{
A Polycyclic Aromatic Hydrocarbon for the Detection of Nonpolar Analytes under Counteracting Humidity Conditions
}

\author{
Nadav Bachar, ${ }^{{ }_{*}}$ Lena Mintz, ${ }^{\perp}+$ Yael Zilberman, ${ }^{\perp}{ }^{+}$Radu Ionescu, ${ }^{\perp}{ }^{\dagger}$ Xinliang Feng, ${ }^{\S}$ \\ Klaus Müllen, ${ }^{\S}$ and Hossam Haick ${ }^{*}, \perp$ \\ $\perp$ The Department of Chemical Engineering and the Russell Berrie Nanotechnology Institute, Technion- \\ Israel Institute of Technology, Haifa 32000, Israel \\ ${ }^{\dagger}$ The Department of Electronics, Electrical and Automatic Engineering, Rovira i Virgili University, 43007 \\ Tarragona, Spain \\ § Max-Planck-Institute for Polymer Research, Postfach 3148, D-55021 Mainz, Germany
}

\begin{abstract}
Real-world samples contain reducing and oxidizing chemical agents as well as large and small (bio)molecules, which are polar and/or nonpolar in nature. Sensing nonpolar analytes, which is of paramount importance for wide variety of applications, is generally more difficult to achieve than sensing polar analytes. Here, we report on empirical observations of a unique polycyclic aromatic hydrocarbon derivative, referred as $\mathrm{PAH}-\mathrm{A}$, whose structure has a triangular-shaped aromatic core (with a carbon number of 60) and contains hydrophobic mesogens terminated with hydrophobic alkyl chains. We show that films made of PAH-A enable excellent sensitivity to nonpolar analytes, compared to polar analytes, in a setting of $5-40 \%$ counteracting relative humidity. This finding is based on monitoring the changes in the physical/optical properties of thin PAH-A films upon exposure to nonpolar and polar analytes, by means of quartz crystal microbalance or spectroscopic ellipsometry measurements. A comparison with other polycyclic aromatic hydrocarbon derivatives with different cores and/or organic functionalities is provided and discussed.
\end{abstract}

\footnotetext{
KEYWORDS: Polycyclic Aromatic Hydrocarbon $\bullet$ Sensor $\bullet$ Detection $\bullet$ Nonpolar $\bullet$ Polar $\bullet \mathrm{Hu}-$ midity
} 


\section{INTRODUCTION}

Real-world samples contain reducing and oxidizing chemical agents as well as large and small (bio)molecules, which are polar and/or nonpolar in nature. ${ }^{1}$ Sensing nonpolar analytes, which serve, amongst the rest, as diagnostic markers of various diseases, ${ }^{2-8}$ is generally more difficult to achieve than sensing polar analytes. ${ }^{1,9,10}$ Polar analytes are usually detected directly through charge exchange between the sensing material and the analyte, ${ }^{1,9,11-13}$ and chemical selectivity of polar analytes is achieved through covalent functionalization with suitable organic molecules that interact strongly with the targeted analyte(s). ${ }^{14-16}$ Nonpolar analytes, on the other hand, have to be detected indirectly via dielectric effects ${ }^{11,12}$ or via steric interaction between the sensing material and the analyte (e.g., film swelling due to analyte adsorption), which might alter the transport of specific physical properties, such as charge carrier or optical wavelength. ${ }^{1,4,5,14}$ Nevertheless, the interaction of the nonpolar analytes with sensing materials under real-world conditions is still hindered by weak to moderate selectivity and, also, by high and/or non-constant humidity levels present in the environment and/or targeted sample. ${ }^{17-20}$

Recently, we have shown that an array of cross-reactive chemiresistive bilayers of single-wall carbon nanotubes and derivatives of polycyclic aromatic hydrocarbon $(\mathrm{PAH}),{ }^{18-20}$ in conjugation with sophisticated pattern recognition methods, provides $>90 \%$ classification accuracy between polar and nonpolar analytes. ${ }^{18}$ While promising, the use of sensor arrays requires increased power consumption and employment of sophisticated and time-consuming pattern recognition algorithms for data analysis and interpretation. A single, standalone sensor that can detect nonpolar analytes under counteracting humidity conditions would have major scientific and technological benefits.

In this paper, we report on a unique PAH derivative, referred as PAH-A, whose structure has a triangular-shaped aromatic core (with a carbon number of 60) and contains hydrophobic mesogens terminated with hydrophobic alkyl chains - see Figure 1 and Table 1. We show that films made of PAH-A enable excellent detection of nonpolar analytes from polar analytes at various counteracting humidity levels. This finding is based on monitoring the changes in the physical/optical properties of PAH-A thin film (see Figure 1 and Table 1) upon exposure to representative nonpolar analytes (decane, octane, hexane, di-butylether and mestylene) and, for the sake of comparison, to polar analytes (decanol, octanol, hexanol and ethanol), under different relative humidity (RH) levels, by means of spectroscopic ellipsometry (SE) and quartz crystal microbalance (QCM) - NOTE: RH is defined as the ratio of the partial pressure of water vapor in the air-water mixture to the saturated vapor pressure of water at 
the prescribed temperature. The unique sensing properties of the PAH-A are compared with those obtained with other PAH derivatives, having carbon corona of different shapes and sizes and terminated with either hydrophobic or hydrophilic functional substituents (PAH-B, PAH-C, and PAH-1 to PAH6 ; $c f$. Figure 1 and Table 1).

\section{EXPERIMENTAL SECTION}

\section{Properties of PAH Derivatives}

Figure 1 and Table 1 summarize the main structural properties of the PAH derivatives used in this study. The synthesis of PAH-A, ${ }^{21} \mathrm{PAH}-\mathrm{B}^{22}, \mathrm{PAH}-\mathrm{C}^{22}, \mathrm{PAH}-1^{23}$, and PAH- $2^{24}$ has been described in previous reports, whereas the synthesis of PAH-3 to PAH-6 will be reported elsewhere. These PAH derivatives contain hydrophobic mesogens that are terminated with different alkyl chains and functional substituents, such as alcohol (for PAH-6), ester (for PAH-2 and PAH-3), carboxylic acid (for PAH-4 and PAH-5), etc. PAH-B, PAH-C, PAH-1, PAH-2 and PAH-6 contain a PAH aromatic core (with a carbon number of 42), while PAH-3, PAH-4 and PAH-5 have a semi-triangle shaped aromatic core (with a carbon number of 48) and PAH-A possesses a triangle-shaped core (with a carbon number of 60). These PAH molecules are able to self-assemble into long molecular stacks with a large, electron rich, semiconducting core, which guarantees good charge carrier transport along the molecular stacking direction and a relatively insulated periphery. ${ }^{21,24-26}$ Furthermore, the nanometer thick PAH columns can easily form 3D, micrometer sized, sponge-like structures with a high surfaceto-volume ratio. ${ }^{20}$ 

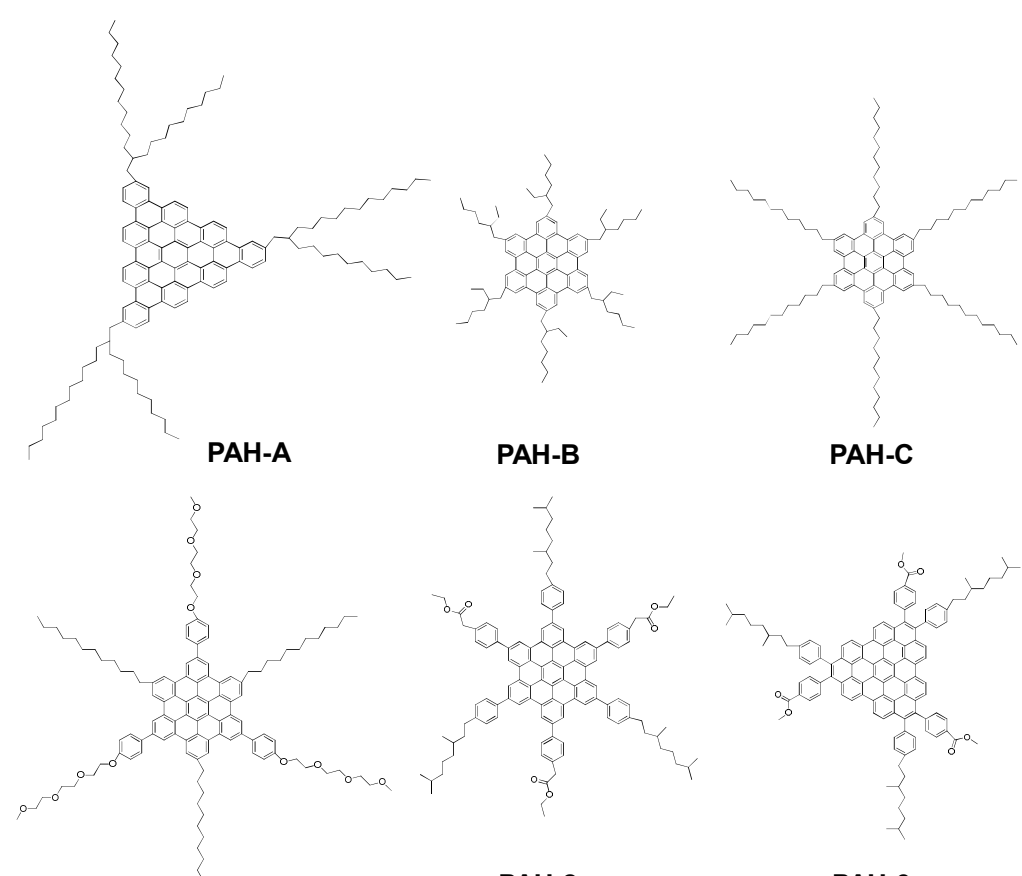

PAH-C

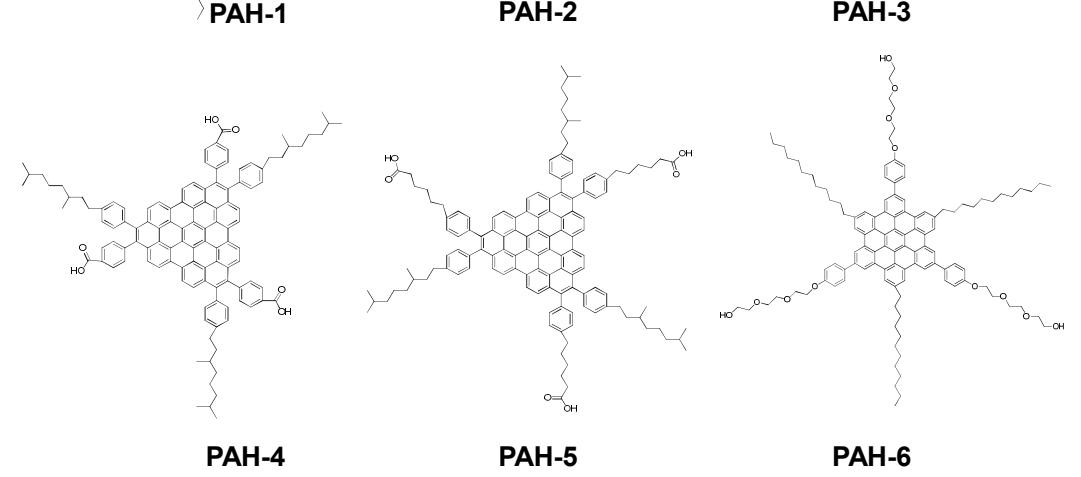

Figure 1. Structures of PAH derivatives used in the current study - $c f$. Table 1.

Table 1. Main Structural Properties of the PAH Derivatives Used in the Experiments

\begin{tabular}{|c|c|c|c|c|}
\hline PAH & Corona Core Shape & $\begin{array}{c}\text { Number of Carbon } \\
\text { Atoms in Corona }\end{array}$ & $\begin{array}{c}\text { Side-Group } \\
\text { Termination }\end{array}$ & Side-Group Type \\
\hline PAH-A & Triangular & 60 & Alkyl Chain & Non-Polar \\
\hline PAH-B & Hexagonal & 42 & Alkyl Chain & Non-Polar \\
\hline PAH-C & Hexagonal & 42 & Alkyl Chain & Non-Polar \\
\hline PAH-1 & Hexagonal & 42 & Ether (methyl) & Weak Polar \\
\hline PAH-2 & Hexagonal & 42 & Ester (ethyl) & Strong Polar \\
\hline PAH-3 & Semi-Triangular & 48 & Ester (methyl) & Strong Polar \\
\hline PAH-4 & Semi-Triangular & 48 & Carboxyl & Strong Polar \\
\hline PAH-5 & Semi-Triangular & 48 & Carboxyl & Strong Polar \\
\hline PAH-6 & Hexagonal & 42 & Alcohol (hydroxyl) & Strong Polar \\
\hline
\end{tabular}




\section{Preparation of PAH Films}

PAH-A, PAH-C, PAH-1, PAH-2, PAH-3 or PAH-6 solutions were prepared at $10^{-3} \mathrm{M}$ concentration in toluene. PAH-4 or PAH-5 solutions were prepared at $10^{-3} \mathrm{M}$ concentration in tetrahydrofuran (THF). PAH-B was prepared at $10^{-3} \mathrm{M}$ concentration in xylene. For Spectroscopic Ellisopmetry (SE) experiments, $100 \mu \mathrm{L}$ PAH solution was drop-casted onto planar p-type $\operatorname{Si}(100)$ substrate that was coated with a thermally grown $100 \mathrm{~nm} \mathrm{SiO} 2$ layer. The samples were then slowly dried under ambient conditions for $30 \mathrm{~min}$ and subsequently placed in a desiccator and kept under vacuum overnight. This process led to the formation of nearly continuous films, with thicknesses ranging between $100 \pm 5 \mathrm{~nm}$ and $800 \pm 10 \mathrm{~nm}$. For Quartz Crystal Microbalance (QCM) experiments, $2 \mu \mathrm{L}$ of PAH solution were drop-casted on gold-plated QCM resonators. The samples were then slowly dried under ambient conditions for $30 \mathrm{~min}$ and subsequently placed in a desiccator and kept under vacuum overnight. The deposition process was repeated twice to completely cover the gold electrode with nearly continuous PAH layers.

\section{Exposure to Analytes}

Oil-free, purified air, obtained from a compressed air source, which had a baseline RH of 5.0 $\pm 0.2 \%$ and an organic contamination of $<0.3 \mathrm{ppm}$ (measured by a commercial PID detector - ppbRAE 3000), was used as a carrier gas for the analytes and as reference gas. The vapours of different analytes or water were produced by bubbling air through their liquid states, using glass bubblers. The analyte saturated vapour was carried out the side arm of the bubbler and diluted with a dilution flow of carrier gas at a flow rate of $0.5-5.01 / \mathrm{min}$. The RH of the dilution flow was regulated by mixing dry $(5.0 \pm 0.2 \% \mathrm{RH})$ and wet $(100 \% \mathrm{RH}$, coming out of the water bubbler) air flows. In this manner, the partial pressures of analyte vapours could be varied between $p_{a} / p_{o}=0.01-0.2$ (where $p_{a}$ stands for the partial pressure of the analyte and $p_{o}$ stands for the saturated vapor pressure at $21^{\circ} \mathrm{C}$ ) in an environment of $5 \% \mathrm{RH}$ to $40 \% \mathrm{RH}$, where humidity effects become seriously significant on the sensors performance. ${ }^{27-33}$ Unless otherwise stated, we focus our presentation on analytes at $p_{a} / p_{o}=0.08$ in an environment of either $5 \%$ RH or $40 \%$ RH.

For testing the effect of ambient humidity on the sensors' responses to the analytes, binary mixtures of volatile analyte and water vapour were used. Sensing experiments with SE and QCM were performed continuously at $20 \pm 2{ }^{\circ} \mathrm{C}$, using three subsequent exposure cycles: (i) exposure to a dry air flow $(5.0 \pm 0.2 \% \mathrm{RH})$ for $10 \mathrm{~min}$; (ii) exposure to the analyte of interest at $p_{a} / p_{o}=0.01-0.2$ in 
$5 \%$ or $40 \% \mathrm{RH}$ background for $10 \mathrm{~min}$; and (iii) purging the chamber with a flow of dry air for 10 min. All measurements were repeated three times in order to check the congruency of the results. The analytes used in the experiments were: DI-water that is provided by a commercial water purification system (Easypure II), 1-decanol (Sigma-Aldrich Ltd), di-butylether (Sigma-Aldrich Ltd), mesitylene (tri-methylbenzene; Sigma-Aldrich Ltd), 1-hexanol (Fluka Analitical Ltd), 1-octanol (Fluka Analitical Ltd), ethanol (Frutarom Ltd), n-hexane (Frutarom Ltd), n-decane (Arcos Organics), and n-octane (Merck Schuchardt Ltd). All analytes had over 99\% purity. All measurements were repeated three times in order to check the congruency of the results. The responses of the SE and QCM were satisfactorily reproducible from sample-to-sample $( \pm 2 \%$ variance) and from batch-to-batch ( $\pm 3 \%$ variance). The PAH films exhibited negligible changes over time ( $\pm 2 \%$ experimental error $)$ - for an extended period of 6-8 months - as well as negligible responses towards fluctuations in temperature $( \pm 2$ ${ }^{\circ} \mathrm{C}$ ) in the measurement apparatus.

\section{Spectroscopic Ellipsometry (SE)}

SE is an optical technique used for studying dielectric properties and changes in thin film thicknesses. These properties are calculated from the measurement of changes in the polarization of the light that was reflected from the analyzed sample. In the current study, reflected light spectra were recorded over the 250-1700 $\mathrm{nm}$ range using a variable angle rotating compensator spectroscopic ellipsometer (M-2000U Automated Angle, J. A. Woollam Co., Inc., USA), equipped with a specially designed triangular exposure cell that allowed in situ measurements at an angle of incidence of $70^{\circ}$. The thickness of the $\mathrm{SiO}_{2}$ layer was determined prior to the deposition of the PAH films at three incidence angles $\left(65^{\circ}, 70^{\circ}, 75^{\circ}\right)$ on an open sample stage, using tabulated values for the refractive indices of $\mathrm{Si}$ and $\mathrm{SiO}_{2}{ }^{34,35}$ A three-phase cap-layer/ $\mathrm{SiO}_{2} / \mathrm{Si}$ model was employed to extract the optical constants and the thickness $(T)$ of the PAH layers, using the effective media approximation to account for the inclusion of voids into the PAH layer. ${ }^{36} \mathrm{SE}$ was employed afterwards to monitor the thickness changes of the PAH layers during exposures to the (gaseous) analytes, assuming that the changes in the optical constants are much smaller than the thickness changes - NOTE: This assumption is based on the similar dielectric properties of the organic analytes and the organic cap-layers. Our SE probe had an area of about $1 \times 5 \mathrm{~mm}^{2}$, and the thickness might vary locally. However, the increase of the average thickness upon exposure to the analytes, measured in the same place of the sample, can be used as a measure for the amount of analytes adsorbed on the PAH films. 


\section{Quartz Crystal Microbalance (QCM)}

QCM is a piezoelectric mass-sensing device that has the ability to measure very small mass changes, down to a fraction of a monolayer or a single layer of atoms. QCM measures a mass per unit area by measuring the change in frequency $(\delta f)$ of a quartz crystal resonator that is disturbed by the addition or removal of a small mass $(\Delta m)$ at the surface of the acoustic resonator. The relationship between $\Delta m$ and $\delta f$ is described by the Sauerbrey's equation:

$$
\Delta m=-C_{f} A \cdot \delta f
$$

where $C_{f}$ is the mass sensitivity $\left(=1.104 \mathrm{ng} \cdot \mathrm{cm}^{-2} \cdot \mathrm{Hz}^{-1}\right)$ and $A$ is the active area of the QCM resonator $\left(=0.2 \mathrm{~cm}^{2}\right) .{ }^{11}$ In the current study, gold-plated QCM resonators $(5 \mathrm{~mm}$ in diameter; $20 \mathrm{MHz}$ resonant frequency) were used to explore changes in the mass of the PAH sensing films caused by exposures to the (gaseous) analytes. Frequency shifts $(\delta f)$, before and after exposure to analytes, were recorded by LibraNose 2.1 (Technobiochip, Elba Island, Italy), which is a system composed of a measurement chamber with eight QCM sensors, an internal micro-pump and a micro-electric valve that conveys the gas samples to the measuring chamber.

\section{RESULTS and DISCUSSION}

Figure 2 presents changes in the SE and QCM signals of PAH-A thin film upon exposure to various polar and nonpolar analytes at $p_{a} / p_{o}=0.08$, in the presence of different $\mathrm{RH}$ conditions in the background. As seen in the figure, exposure of the PAH-A films to various analytes leads to abrupt changes in the SE signals (changes in the thickness of the films - see Experimental Section). The SE signals stabilize quickly (within 1-2 sec) and maintain almost constant value during the whole exposure period. This behavior is observed regardless the type of the analyte. As for the QCM responses (changes in the adsorbed mass of analytes in/on the films - see Experimental Section), all nonpolar analytes (excluding hexane) lead to slow changes in the sensing signal after exposure, indicating an increase in the mass of adsorbed analyte in/on the PAH-A film. In the case of hexane, a very rapid change in film's mass is produced immediately after the exposure. However, following the rap$\mathrm{id} /$ initial response, a continuous (slight) decrease in the response is obtained. Further securitization of 
the results presented in Figure 2 shows that the nonpolar analytes exhibit significantly higher signals than the polar analytes for both SE and QCM platforms.

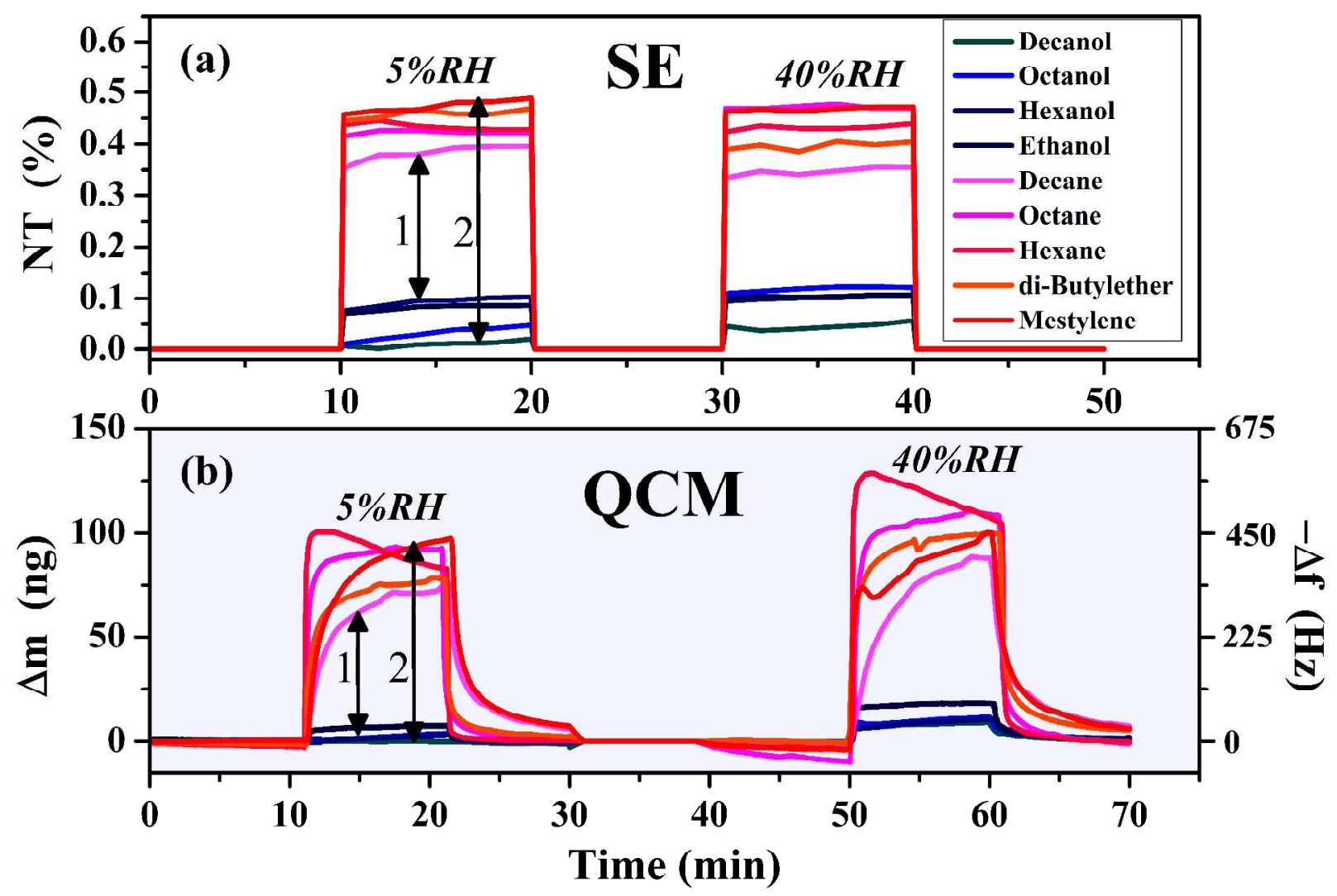

Figure 2. (a) Thickness changes of the PAH-A film measured by $\mathrm{SE}\left(N T=\Delta T / T_{0}\right.$, where $\Delta T$ stands for the change in the thickness and $T_{0}$ stands for the film's thickness before exposure) upon exposure to various polar and nonpolar analytes in the presence of different humidity contents. (b) Changes in frequency (- $\Delta f$; right $\mathrm{y}$ axis) of a QCM that is disturbed by the addition or removal of a small mass ( $\Delta m$; left y-axis) at the surface of the acoustic resonator upon exposure to various polar and nonpolar analytes in the presence of different humidity contents. ${ }^{10}$ The polar analytes are represented in green and blue, while the nonpolar analytes are represented in red, orange and pink. The gap between the signals acquired in the presence of nonpolar and polar analytes $\left(G_{n p-p}\right)$ is presented by Arrow 1 and the range of responses (RR) is presented by Arrow 2. $G_{n p-p}$ was calculated as lowest response to any nonpolar analyte minus highest response to any polar analyte, while $R R$ as highest response to any of the analytes minus lowest response to any of the analytes. 
From Figure 2, we could calculate important parameters associated with the detection ability of nonpolar analytes, such as the gap between the signals acquired in the presence of nonpolar and polar analytes $\left(G_{n p-p}\right)$ and the spread of the signals acquired in the presence of analytes of similar nature $\left(S_{n p}\right.$ and $S_{p}$ for nonpolar and polar analytes, respectively). The extracted values (Table 2) show that $G_{n p-p}$ is much higher (1.9 to 8.2 times higher) than either $S_{n p}$ or $S_{p}$. The values from Table 2 obtained by QCM are similar at both 5\% and 40\% RH, demonstrating the suitability of the PAH-A for the detection of nonpolar analytes in the presence of counteracting background of 5\% to $40 \% \mathrm{RH}$. Similar observations are obtained by the SE, with one main difference: the sensing signals acquired by $\mathrm{SE}$ technique at $40 \% \mathrm{RH}$ are slightly higher (15-18\%) than the sensing signals acquired at $5 \%$ RH.

Table 2. Gap between the signals provided by nonpolar and polar analytes exposures $\left(G_{n p-p}\right)$, and spread of the signals acquired upon exposure to nonpolar analytes of similar nature $\left(S_{n p}\right)$ and polar analytes of similar nature $\left(S_{p}\right)$.

\begin{tabular}{|l|c|c|c|c|c|c|}
\hline & $R H(\%)$ & $\mathrm{G}_{\text {np-p }}$ & $\mathrm{S}_{\text {np }}$ & $\mathrm{S}_{\mathrm{p}}$ & $\mathrm{G}_{\text {np-p }} / \mathrm{S}_{\text {np }}$ & $\mathrm{G}_{\text {np-p }} / \mathrm{S}_{\mathrm{p}}$ \\
\hline \multirow{2}{*}{$\mathrm{SE}(\%)$} & 5 & $0.29 \pm 0.05$ & $0.09 \pm 0.02$ & $0.08 \pm 0.01$ & $3.22 \pm 0.48$ & $3.61 \pm 0.54$ \\
\cline { 2 - 7 } & 40 & $0.23 \pm 0.04$ & $0.12 \pm 0.02$ & $0.06 \pm 0.01$ & $1.89 \pm 0.29$ & $3.80 \pm 0.57$ \\
\hline \multirow{2}{*}{ QCM (ng) } & 5 & $65.9 \pm 0.4$ & $23.0 \pm 0.4$ & $7.8 \pm 0.4$ & $2.8 \pm 0.1$ & $8.2 \pm 0.1$ \\
\cline { 2 - 7 } & 40 & $68.1 \pm 0.4$ & $24.1 \pm 0.4$ & $9.2 \pm 0.4$ & $2.8 \pm 0.1$ & $7.5 \pm 0.1$ \\
\hline
\end{tabular}

Intuitively, the excellent sensitivity of PAH-A towards nonpolar analytes under high counteracting humidity levels $(40 \% \mathrm{RH})$ could be ascribed to the hydrophobic nature of the film. To examine this hypothesis, the sensing properties of PAH-A were compared with other hydrophobic PAH derivatives (PAH-B and PAH-C) as well as with a series of more hydrophilic PAH derivatives (PAH-1 to PAH-6). PAH-B and PAH-C contain hydrophobic mesogens terminated with hydrophobic alkyl chains similar in their chemical nature/structure to those of PAH-A, while derivatives 1 to 6 contain also mesogans terminated with hydrophilic functional groups such as hydroxyl and carboxyl - see Figure 1 and Table 1. Figure 3 compares the sensing properties of PAH-A with PAH-B and PAH-C as well as the sensing properties of PAH-A with those of PAH-1 to PAH-6. As seen in the figure, the 
signals acquired by PAH-B, PAH-C and PAH-1 to PAH-6 in the presence of nonpolar analytes are in general slightly higher than the signals acquired in the presence of polar analytes. However, in most cases, the $G_{n p-p}$ values are insignificant or even overlapping to each other. As a matter of fact, only QCM-coated PAH-4 could lead to an obvious distinction between the nonpolar and polar analytes. However, increasing the background humidity from $5 \%$ to $40 \% \mathrm{RH}$ increases the $G_{n p-p}$ by $133 \%$ and the $S_{p}$ by $151 \%$ (see Figures $3 \mathrm{c}$ and $3 \mathrm{~d}$ ). This observation indicates that, in contrast to PAH-A/QCM, the PAH-4/QCM is highly affected by the actual humidity background. Furthermore, the non-additive nature of this humidity effect is difficult to compensate by post-treatment processes. ${ }^{17}$ 

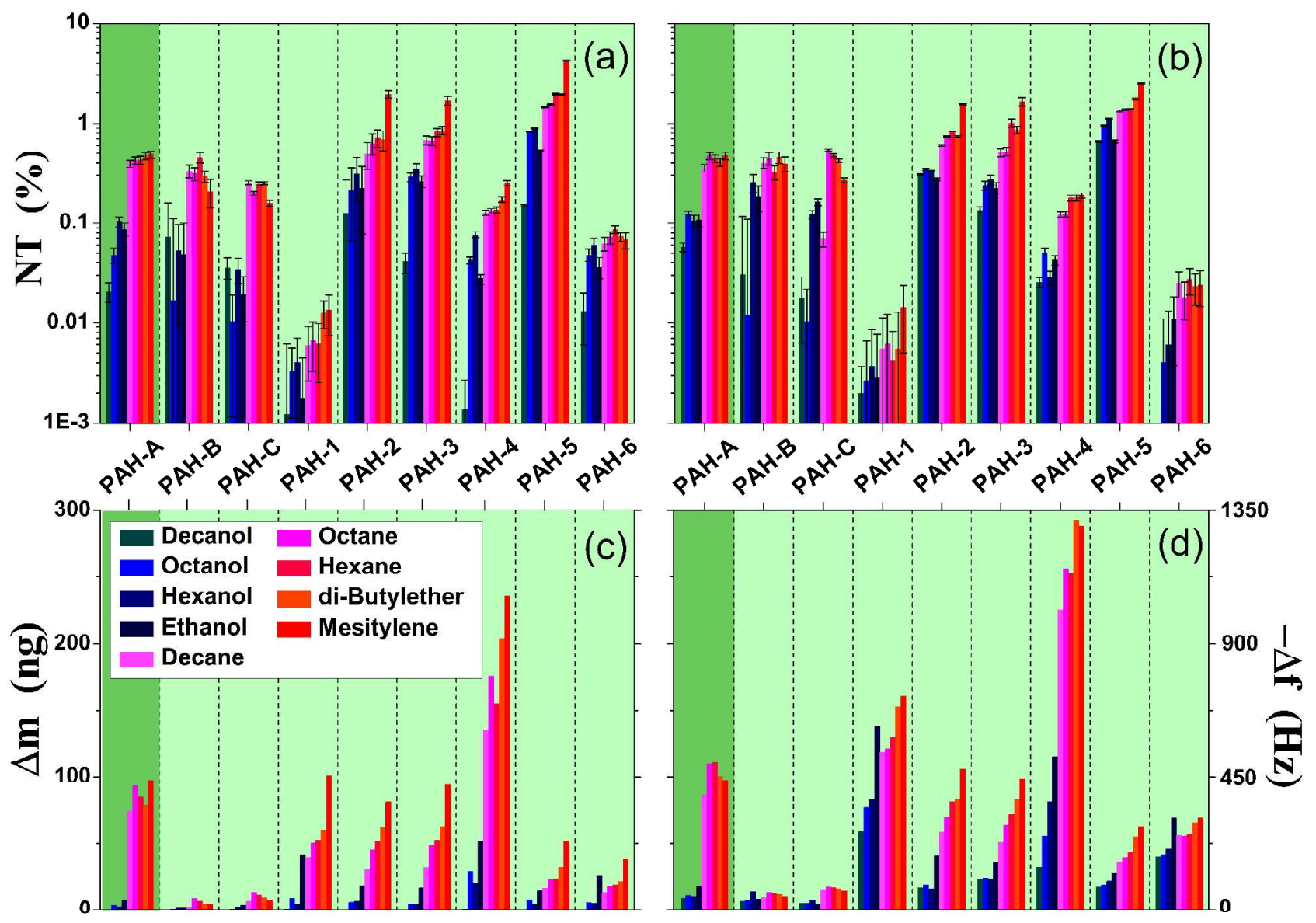

Figure 3. Thickness changes of the various PAH layers ( $c f$. Figure 1) measured by SE $\left(N T=\Delta T / T_{0}\right.$, where $\Delta T$ stands for the change in the thickness and $T_{0}$ stands for the film's thickness before exposure) upon exposure to various polar and nonpolar analytes (a) $5 \% \mathrm{RH}$, and (b) $40 \% \mathrm{RH}$; and changes in frequency (- $\Delta$; ; right $\mathrm{y}$-axis) of a QCM that is disturbed by the addition or removal of a small mass ( $\Delta m$; left y-axis) at the surface of the acoustic resonator upon exposure to various polar and nonpolar analytes at (c) $5 \% \mathrm{RH}$, and (d) $40 \%$ $\mathrm{RH} .{ }^{10}$ The polar analytes are represented in green and blue, while the nonpolar analytes are represented red, orange and pink. For SE measurements, the error bars for the three repetitions are shown. The error bars in the QCM measurements are not shown because they are negligible ( $\pm 1 \mathrm{~Hz}$ or $\pm 0.2208 \mathrm{ng}$ ). Representative reference responses, viz. SE and QCM responses upon exposure to various RH levels without analytes, are presented in the Supporting Information, Figure 1S. 
Table 3. The Ratio between $D A$ for PAH-A and all other PAH films, based on the results obtained in both SE and QCM studies.

\begin{tabular}{|c|c|c|c|c|c|c|c|c|c|}
\hline & $\overline{\underline{x}}$ & 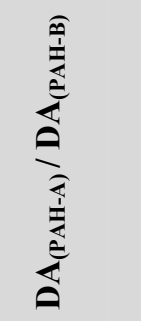 & 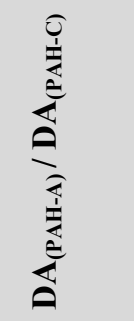 & 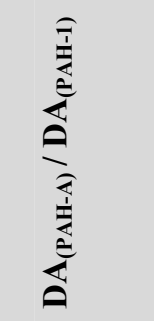 & 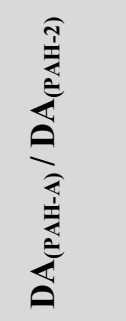 & 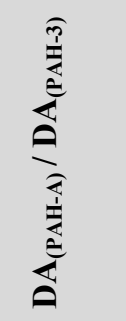 & 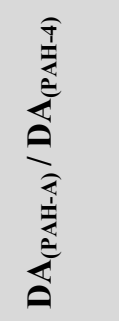 & 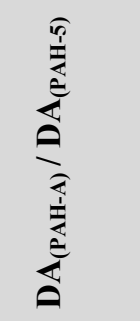 & 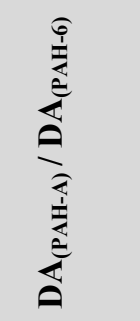 \\
\hline \multirow{2}{*}{ SE } & $5 \%$ & $1.1 \pm 0.2$ & $0.9 \pm 0.1$ & $4.0 \pm 0.6$ & $6.1 \pm 0.9$ & $3.3 \pm 0.5$ & $3.1 \pm 0.5$ & $4.5 \pm 0.7$ & $23.3 \pm 3.5$ \\
\hline & $40 \%$ & $3.7 \pm 0.6$ & $-3.2 \pm 0.5$ & $12.5 \pm 1.9$ & $2.8 \pm 0.4$ & $3.5 \pm 0.5$ & $1.3 \pm 0.2$ & $4.6 \pm 0.7$ & $2.2 \pm 0.3$ \\
\hline \multirow{2}{*}{ QCM } & $5 \%$ & $26.6 \pm 3.5$ & $3.0 \pm 0.6$ & $-40.8 \pm 5.9$ & $4.8 \pm 0.3$ & $4.3 \pm 0.2$ & $1.9 \pm 0.1$ & $17.1 \pm 5.3$ & $-2.0 \pm 0.1$ \\
\hline & $40 \%$ & $-0.9 \pm 0.1$ & $1.2 \pm 0.1$ & $-3.5 \pm 0.1$ & $3.5 \pm 0.1$ & $3.4 \pm 0.2$ & $1.6 \pm 0.1$ & $3.2 \pm 0.2$ & $-1.4 \pm 0.1$ \\
\hline
\end{tabular}

The ability of the PAH films to discriminate between nonpolar and polar analytes were examined further by dividing the $G_{n p-p}$ by the range of responses $(R R=$ highest response to any of the analytes minus lowest response to any of the analytes, i.e., the total spread of the responses to all analytes analyzed), viz.:

$$
D A=\frac{G_{n p-p}}{R R}
$$

Table 3 shows the ratio between $D A$ calculated for PAH-A and all other PAH films considered in this study (i.e., PAH-B, PAH-C, and PAH-1 to PAH-6). The fact that almost all values from Table 3 are $>|1|$, independent of the measurement method and/or background humidity, unequivocally demonstrates that PAH-A is the most suitable material for clear differentiation between the nonpolar and polar analytes - NOTE: the negative values in Table 3 indicate overlapping between the responses to nonpolar and polar analytes in these cases. The excellent sensitivity of the PAH-A might be ascribed to the preferred dissolution of the nonpolar analytes into the PAH-A film, in comparison to polar analytes. $^{37,38}$ The preferred analyte dissolution leads to higher swelling effect (i.e., an increase in the PAH-A films' thickness) upon exposure to nonpolar analytes, compared to polar analytes. ${ }^{19}$ The low dissolution of the water molecules in the PAH-A's hydrophobic side-terminations prevent the water penetration into the PAH-A film. However, the QCM results demonstrate that the water molecules 
exist on the PAH-A surface, most probably as a condensed layer. For each analyte, the mass adsorbed on the PAH-A/QCM (proportional to the measured $\Delta m$ or $-\Delta f$; see Experimental Section) at $40 \% \mathrm{RH}$ was higher than that adsorbed at 5\% RH (Figure 2). Additionally, the difference between the QCM response at $40 \% \mathrm{RH}$ and $5 \% \mathrm{RH}$ was almost constant (within $\pm 8 \%$ experimental error) and equivalent to the mass of water layer condensed at the PAH-A/QCM surface. With this in mind, it is reasonable to argue that water molecules are adsorbed on the PAH-A surface, but do not affect the SE signals, due to negligible changes in the PAH-A film thickness and/or refractive index.

Measuring the SE and QCM responses of the PAH-A films upon exposure to different analyte concentrations (see Experimental Section) revealed a detection limit of 1-10 ppm. This range of detection limits is equivalent to the majority of sensing technologies, but, on the other hand, is lower than other state-of-the-art ones. For example, state-of-the-art nanomechanical oscillators have high detection limits (down to sub-ppb levels), ${ }^{39,40}$ but the high costs of fabrication and high-vacuum and low-temperatures required for operation make them currently impracticable to some extent. Additionally, there is a substantial possibility that the high levels of RH neutralize the frequency (changes) produced by the nanomechanical oscillators. Chemiresistors of conducting polymers are simple to implement but the high response to humidity (also, when the humidity coexist in mixtures containing $1-10$ s ppm of nonpolar VOCs) can be disadvantage. ${ }^{41,42}$ Chemicapacitors are more stable than chemiresistors, but the response to humidity as well as the long times of response and recovery might be a disadvantage. ${ }^{43}$ Polymer-coated surface acoustic wave $(\mathrm{SAW})^{44}$ or $\mathrm{QCM}^{45}$ sensors are mostly responsive to humidity and, also, limited to a detection level of few ppm.

\section{SUMMARY AND CONCLUSIONS}

The results presented in this study evidence excellent detection of nonpolar analytes achieved with a gas-sensitive film made of PAH-A. Even though the results presented here were obtained through empirical observations, we believe that they are extremely useful for important practical applications under counteracting humidity conditions in the range between 5\% and $40 \% \mathrm{RH}$. As yet, the origin of the sensing mechanism of the studied PAH-A derivative is still puzzling. Studies investigating the sensing properties of PAH molecules whose mesogen's shape and size as well as functional groups are, synthetically, changed systematically, would provide an insight on the sensing mechanism of the PAH-A. Since the hydrophobicity per se cannot explain the obtained results (mainly PAH-A versus PAH-B and PAH-C), studying the self-assembly and morphological properties of 
the PAH-A is of critical importance. Elucidating the PAH-A discriminative power towards nonpolar analytes under higher humidity $(40-100 \% \mathrm{RH})$ conditions, where the interference of water molecules is likely higher than the 5-40\% RH region, ${ }^{27-33}$ would widen the spectrum of applications of this material under counteracting humidity conditions in the future. The method is simple to apply and interpret, and does not require the use of sophisticated pattern recognition algorithms.

\section{ASSOCIATED CONTENT}

Supporting Information. Exposure of PAH-A, PAH-C, and PAH-4 to variable humidity levels, without analytes. This material is available free of charge via the Internet at http://pubs.acs.org.

\section{AUTHOR INFORMATION}

$\$$ These authors contributed equally to the paper.

\section{Corresponding Author}

hhossam@technion.ac.il

\section{ACKNOWLEDGMENT}

The research leading to these results has received funding from the FP7-Health Program under the LCAOS (grant agreement no. 258868), the FP7's ERC grant under DIAG-CANCER (grant agreement no. 256639), DFG Priority Program SPP 1355, ESF Project GOSPEL (grant agreement no. 09-EuroGRAPHENE-FP-001), EU Project GENIUS, and ERC grant on NANOGRAPH. We thank Dr. Ulrike (Mirjam) Tisch (Technion IIT) for her help in performing and interpreting the SE experiments and A'laa Garaa (Technion IIT), Fairouz Muallem (Galilee Christian High School, Eilaboun), Gady Konvolina (Technion IIT), and Lucy Liberman (Technion - IIT) for assistance. 


\section{REFERENCE}

(1) Tisch, U.; Haick, H. MRS Bull. 2010, 35, 797-803.

(2) Tisch, U.; Billan, S.; Ilouze, M.; Phillips, M.; Peled, N.; Haick, H. CML - Lung Cancer 2012, 5, $107-$ 117.

(3) Tisch, U.; Haick, H. Rev. Chem. Eng. 2010, 26, 171-179.

(4) Peng, G.; Tisch, U.; Adams, O.; Hakim, M.; Shehada, N.; Broza, Y. Y.; Billan, S.; Abdah-Bortnyak, R.; Kuten, A.; Haick, H. Nature Nanotech. 2009, 4, 669-673.

(5) Peng, G.; Tisch, U.; Haick, H. Nano Lett. 2009, 9, 1362-1368.

(6) Ionescu, R.; Broza, Y.; Shaltieli, H.; Sadeh, D.; Zilberman, Y.; Feng, X.; Glass-Marmor, L.; Lejbkowicz, I.; Müllen, K.; Miller, A.; Haick, H. ACS Chem. Neurosci. 2011, 2, 687-693.

(7) Barash, O.; Peled, N.; Tisch, U.; Bunn, P. A.; Hirsch, F. R.; Haick, H. Nanomedicine (New York, NY, United States) 2012, 8, 580-589.

(8) Barash, O.; Peled, N.; Hirsch, F. R.; Haick, H. Small 2009, 5, 2618-24.

(9) Rock, F.; Barsan, N.; Weimar, U. Chem. Rev. 2008, 108, 705-725.

(10) Dickert, F. L.; Geiger, U.; Lieberzeit, P.; Reutner, U. Sens. Actuat. B 2000, 70, 263-269.

(11) Paska, Y.; Stelzner, T.; Assad, O.; Tisch, U.; Christiansen, S.; Haick, H. ACS Nano 2012, 6, 335-345.

(12) Paska, Y.; Haick, H. ACS Appl. Mater. Interf. 2012, 4, 2604-2617.

(13) Paska, Y.; Stelzner, T.; Christiansen, S.; Haick, H. ACS Nano 2011, 5, 5620-5626.

(14) Haick, H. J. Phys. D 2007, 40, 7173-7186.

(15) Angione, M. D.; Pilolli, R.; Cotrone, S.; Magliulo, M.; Mallardi, A.; Palazzo, G.; Sabbatini, L.; Fine, D.; Dodabalapur, A.; Cioffi, N.; Torsi, L. Mater. Today 2011, 14, 424-433.

(16) Crowe, L. L.; Solntsev, K. M.; Tolbert, L. M. Langmuir 2007, 23, 6227-6232.

(17) Konvalina, G.; Haick, H. ACS Appl. Mater. Interf. 2012, 4, 317-325

(18) Zilberman, Y.; Ionescu, R.; Feng, X.; Mullen, K.; Haick, H. ACS Nano 2011, 5, 6743-6753

(19) Zilberman, Y.; Tisch, U.; Shuster, G.; Pisula, W.; Feng, X.; Mullen, K.; Haick, H. Adv. Mater. 2010, $22,4317-4320$.

(20) Zilberman, Y.; Tisch, U.; Pisula, W.; Feng, X.; Mullen, K.; Haick, H. Langmuir 2009, 25, 5411-5416.

(21) Feng, X. L.; Liu, M. Y.; Pisula, W.; Takase, M.; Li, J. L.; Müllen, K. Adv. Mater. 2008, 14, 2684-2689.

(22) Kastler, M.; Pisula, W.; Wasserfallen, D.; Pakula, T.; Müllen, K. J. Am. Chem. Soc. 2005, 127, 42864296.

(23) Stabel, A.; Herwig, P.; Müllen, K.; Rabe, J. P. Angew. Chem. Int. Ed. 1995, 34, 1609-1611.

(24) Feng, X. L.; Pisula, W.; Zhi, L. J.; Takase, M.; Müllen, K. Angew. Chem. Int. Ed. 2008, 47, 1703-1706.

(25) Feng, X. L.; Pisula, W.; Kudernac, T.; Wu, D. Q.; Zhi, L. J.; De Feyter, S.; Müllen, K. J. Am. Chem. Soc. 2009, 131, 4439-4448.

(26) Feng, X. L.; Marcon, V.; Pisula, W.; Hansen, M. R.; Kirkpatrick, J.; Andrienko, D.; Kremer, K.; Müllen, K. Nature Mater. 2009, 8, 421-426.

(27) Yan, H.-Q.; Liu, C.-C. Sens. Actuat. B 1993, 10, 133-136.

(28) Zarnik, M. S.; Belavic, D. Metrol. Meas. Syst. . 2012, XIX, 133-140.

(29) Bouhadid, M.; Redon, N.; Plaisance, H.; Desbrieres, J.; Reynaud, S. Macromol. Symp. 2008, 268, 9-13.

(30) Bai, Z.; Xiea, C.; Hu, M.; Zhang, S.; Zeng, D. Mater. Sci. Eng. 2008, 149, 12-17.

(31) Belghachi, A.; Collins, R. A. J. Phys. D 1990, 23, 223-227.

(32) Eaton, K.; Douglas, P. Sens. Actuat. B 2002, 82, 94-104.

(33) Vlachos, D. S.; Skafidas, P. D.; Avaritsiotis. Sens. Actuat. B 1995, 24-25, 491-494.

(34) Aspnes, D. E.; Theeten, J. B. J. Electrochem. Soc. 1980, 127, 1359-1365.

(35) Philipp, H. R., Handbook of Optical Constants of Solids. Academic Press, Inc.: Orlando, Florida, 1985.

(36) Leinfellner, N.; Ferré-Borrull, J.; Bosch, S. Microelect. Rel. 2000, 40, 873-875.

(37) Bennett, M. E.; Alexander, W. A.; Lu, J. W.; Troya, D.; Morris, J. R. J. Phys. Chem. C 2008, 112 , 17272-17280.

(38) Li, B.; Lambeth, D. N. Nano Lett. 2008, 8, 3563-3567.

(39) Ekinci, K. L.; Huang, X. M. H.; Roukes, M. L. Appl. Phys. Lett. 2004, 84, 4469-4471. 
(40) Ekinci, K. L.; Yang, Y. T.; Roukes, M. L. J. Appl. Phys. 2004, 95, 2682-2689.

(41) Gardner, J. W.; Bartlett, P. N., Electronic noses: principles and applications. Oxford University Press: Oxford, 1999.

(42) Faglia, G.; Bicelli, F.; Sberveglieri, G.; Maffezzoni, P.; Gubian, P. Sens. Actuat. B 1997, B44, 517-520.

(43) Patel, S. V.; Mlsna, T. E.; Fruhberger, B.; Klaassen, E.; Cemalovic, S.; Baselt, D. R. Sens. Actuat. B 2003, B96, 541-553.

(44) Yu, H.; Xu, L.; Cao, M.; Chen, X.; Wang, P.; Jiao, J.; Wang, Y. Proc. IEEE Sens. 2003, 2, 1333-1337.

(45) Di Natale, C.; Macagnano, A.; Martinelli, E.; Paolesse, R.; D'Arcangelo, G.; Roscioni, C.; FinazziAgro, A.; D'Amico, A. Biosens. Bioelectron. 2003, 18, 1209-1218. 
Page 17 of 17

1
2
3
4
5
6
7
8
9
10
11
12
13
14
15
16
17
18
19
20
21
22
23
24
25
26
27
28
29
30
31
32
33
34
35
36
37
38
39
40
41
42
43
44
45
46
47
48
49
50
51
52
53
54
55
56
57
58
59
60
ACS Applied Materials \& Interfaces

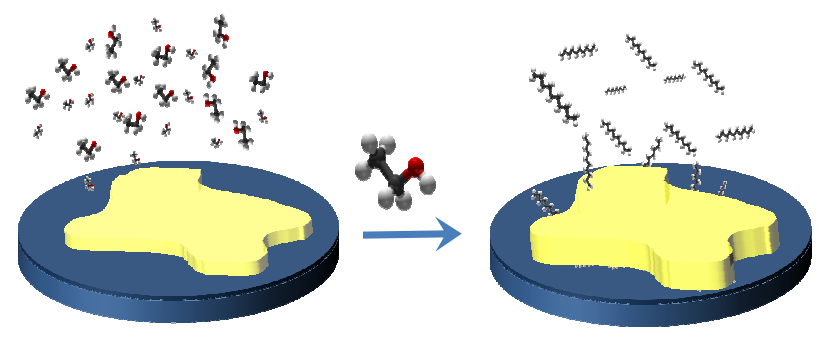

17

ACS Paragon Plus Environment 